\title{
Peripheral Neuropathy as a Risk Factor for Developing Cardiovascular Disease in Diabetic Patients
}

\author{
Ghassan AlGhamdi ${ }^{1}$, Hisham Saati ${ }^{1}$, Enad Almotairi ${ }^{1}$, Basil S. Alsofiani ${ }^{2}$, Abdulhalim J. Kinsara ${ }^{3}$ \\ 1. Medicine, King Saud Bin Abdulaziz University for Health Sciences College of Medicine, Jeddah, SAU 2. Internal \\ Medicine, King Saud Bin Abdulaziz University for Health Sciences College of Medicine, Jeddah, SAU 3. Cardiology, \\ Ministry of National Guard - Health Affairs, King Saud Bin Abdulaziz University for Health Sciences, College of \\ Medicine, Western Region (COM-WR) King Abdullah International Medical Research Center, Jeddah, SAU
}

Corresponding author: Abdulhalim J. Kinsara, akinsara@yahoo.com

\section{Abstract \\ Background}

Type 2 diabetes mellitus (DM) is prevalent in developing countries and is associated with many comorbidities, including diabetic peripheral neuropathy (DPN) and cardiovascular disease (CVD). In this study, we assessed and evaluated the association between DPN and CVD.

\section{Methods}

The study is a cross-sectional study that included DM patients who are attending DM primary care clinics. We evaluated each patient and collected epidemiological data, the physical examination findings, including cardiovascular status, and the presence of DPN. DPN was assessed with the neuropathic disability score (NDS), and it was considered present if the score was 5 or higher. The age and the levels of blood glucose, $\mathrm{HbA1C}$, and plasma total cholesterol were recorded.

\section{Results}

The study included $116 \mathrm{DM}$, the mean age was $64.5 \pm 15$ years (19 to 84 years) and the majority (61.2\%) of the sample were male. The sample was divided into two groups: patients with DM only and patients with DM and CVD. The CVD group had a higher NDS score compared to the non-CVD group $(\mathrm{P}=0.006)$. The result indicated that for the CVD group, the prevalence of DPN was higher (50.8\%) as compared to the non-CVD group (30.2\%) $(\mathrm{P}=0.041)$. DPN was also associated with the acute coronary syndrome (ACS) $(\mathrm{P}=0.013)$ but not heart failure $(\mathrm{HF})(\mathrm{P}=0.427)$. In addition, the $\mathrm{HbA1C}$ was significantly higher in patients with DPN $(\mathrm{P}=0.0345)$.

\section{Conclusion}

Review began $11 / 23 / 2020$ Review ended 11/28/2020 Published 12/05/2020

\section{(c) Copyright 2020}

AlGhamdi et al. This is an open access article distributed under the terms of the Creative Commons Attribution License CC-BY 4.0., which permits unrestricted use, distribution, and reproduction in any medium, provided the original author and source are credited.

Our findings indicate that DPN was more prevalent in the CVD group and, in particular, in the group with ACS. The evidence provides support for the association between DPN and CVD.

Categories: Cardiology, Endocrinology/Diabetes/Metabolism, Neurology

Keywords: diabetes mellitus, diabetic peripheral neuropathy, cardiovascular disease, neuropathic disability score, saudi arabia

\section{Introduction}

Type 2 diabetes mellitus (DM) is prevalent globally, and in Saudi Arabia, the prevalence rate is one of the highest [1]. According to the World Health Organization, the prevalence of diabetes in the total population of Saudi Arabia is $14.4 \%$ [2]. Diabetic peripheral neuropathy (DPN) is a frequent complication associated with DM. DPN is defined as "the presence of symptoms and/or signs of peripheral nerve dysfunction in people with DM after the exclusion of other causes [3]. Another complication of DM is cardiovascular disease (CVD). A study reported that DM increases the chance of developing CVD two-fold, specifically acute coronary syndrome (ACS) or heart failure (HF) [4].

We propose that the shared pathophysiological mechanisms will result in a close association between the two complications. There are only a few studies available regarding the association between DPN and CVD. A study in 2014, conducted in the United Kingdom with 13,043 individuals, reported an association between patients with DPN and an increased risk of CVD [5]. No local studies are available regarding this subject.

The research will provide additional evidence of an association between CVD in DM patients with DPN. 


\section{Cureus}

\section{Materials And Methods}

The study was a cross-sectional study with a sample of 116 DM patients, all over 18 years old and irrespective of gender. Patients with one of the following risk factors were excluded from the study: connective tissue disorders, alcoholism, vitamin B12/folate deficiencies, and primary neurologic disorders (previous spinal injury, a history of lumbar or cervical disc disease, carpal tunnel syndrome, and inherited neuropathy).

The study was approved by the local institutional review board. A standardized data collection form was completed after obtaining written informed consent from the participant. The form collected data about the participant's age, gender, blood pressure, blood glucose, glycated hemoglobin (HbA1C), plasma total cholesterol, low-density lipoprotein, and the presence of CVD with its type.

DPN was recorded as a neuropathy disability score (NDS). An NDS score of 5 or more was indicative of DPN. Sensory, motor, vibration, and temperature neuropathies were assessed. The data was stored on Microsoft Excel (Microsoft Corporation, Redmond, WA) and analyzed with the Statistical Package for the Social Sciences (SPSS) version 22 (IBM Corp., Armonk, NY). DPN was the independent variable, and the outcome variable was the proportion with CVD. The qualitative variables are presented as frequency and percentage, and the quantitative variables as mean and standard deviation, if normally distributed, or if skewed, the median and 25 th to 75 th percentile. A chi-square test was used for the qualitative variables and a MannWhitney $\mathrm{U}$ for quantitative variables. A P-value of $<0.05$ was considered significant, with a confidence interval (CI) of $95 \%$.

\section{Results}

In total, 116 participants who met the inclusion criteria were enrolled in this study, with the majority (61.2\%) male. The mean age was $64.5 \pm 15$ years (19 to 84 years). The sample was assigned to two groups: a group with DM and CVD (49.2\%) and the non-CVD group.

The overall DPN prevalence rate was $41.4 \%$. The CVD group had a significantly higher NDS score $(6 \pm 5)$ as compared to the non-CVD group $(6 \pm 1)(\mathrm{P}=0.006)$. The results indicated that the prevalence of DPN in the CVD group was (50.8\%) as compared to the non-CVD group (32.2\%) ( $\mathrm{P}=0.041)$ (Table 1$)$.

\begin{tabular}{|c|c|c|c|c|}
\hline & \multicolumn{2}{|c|}{ DM with CVD (n=57) } & DM without CVD (n=59) & P-value \\
\hline Age (years) & \multicolumn{2}{|c|}{$65 \pm 16$} & $64 \pm 15$ & 0.21 \\
\hline Gender: Male & 37 & $64.9 \%$ & 34 & 4.51 \\
\hline Acute coronary syndrome & 38 & $63.2 \%$ & 0 & $>0.001$ \\
\hline Heart failure & 26 & $45.6 \%$ & 0 & $>0.001$ \\
\hline Neuropathy disability score (NDS) & \multicolumn{2}{|c|}{$6 \pm 5$} & $6 \pm 1$ & 0.006 \\
\hline Diabetic peripheral neuropathy & 29 & $50.8 \%$ & 19 & 0.041 \\
\hline Blood glucose & \multicolumn{2}{|c|}{$8.7 \pm 7.6$} & $8.8 \pm 3.5$ & 0.987 \\
\hline Glycated hemoglobin (HbA1C) $(\mathrm{mmol} / \mathrm{mol})$ & \multicolumn{2}{|c|}{$7.7 \pm 1.7$} & $8.3 \pm 1.5$ & 0.006 \\
\hline Low-density lipoprotein (LDL) (mg/dL) & \multicolumn{2}{|c|}{$2 \pm 0.8$} & $2.3 \pm 1.2$ & 0.37 \\
\hline Total cholesterol & \multicolumn{2}{|c|}{$3.5 \pm 1.5$} & $4 \pm 1.5$ & 0.01 \\
\hline
\end{tabular}

\section{TABLE 1: Comparison between DM with/without CVD}

DM: diabetes mellitus; CVD: cardiovascular disease

We also found a significant association between the presence of ACS and the development of DPN $(\mathrm{P}=0.013)$, representing 58.33\% of the group with DPN. However, HF had no significant association with DPN $(\mathrm{P}=0.427)$. A higher HbA1c score was associated with a higher prevalence of DPN ( $\mathrm{P}=0.0345)$, (Tables $2-3)$. 


\section{Cureus}

\begin{tabular}{|c|c|c|c|c|}
\hline \multirow{2}{*}{ Variables } & \multicolumn{3}{|c|}{ DPN } & \multirow{2}{*}{ P-value } \\
\hline & \multicolumn{2}{|c|}{ Yes $(n=48)$} & \multirow[t]{2}{*}{ No $(n=68)$} & \\
\hline Gender: Male & 32 & $45.07 \%$ & & 0.311 \\
\hline Age (years) & \multicolumn{2}{|c|}{$64.9 \pm 9.0$} & $59.6 \pm 14.9$ & 0.0610 \\
\hline Acute coronary syndrome & 21 & $58.33 \%$ & 15 & 0.013 \\
\hline Heart failure & 9 & $34.62 \%$ & 17 & 0.427 \\
\hline Blood glucose & \multicolumn{2}{|c|}{$11.2 \pm 4.9$} & $9.15 \pm 3.9$ & 0.0127 \\
\hline Glycated hemoglobin (HbA1c) (mmol/mol) & \multicolumn{2}{|c|}{$8.4 \pm 1.8$} & $7.7 \pm 1.4$ & 0.0345 \\
\hline Low-density lipoprotein (LDL) & \multicolumn{2}{|c|}{$2.3 \pm 0.8$} & $2.3 \pm 0.9$ & 0.9263 \\
\hline Total cholesterol & \multicolumn{2}{|c|}{$3.9 \pm 0.96$} & $3.9 \pm 1.2$ & 0.7922 \\
\hline
\end{tabular}

TABLE 2: Characteristics of DM patients with DPN

DM: diabetes mellitus; DPN: diabetic peripheral neuropathy

\begin{tabular}{|c|c|c|c|}
\hline Variable & $D P N+D M+C V D(n=29)$ & DPN +DM (n=19) & P-value \\
\hline Gender: Male & 18 & 14 & 0.404 \\
\hline Age & $65.2 \pm 8.4$ & $64.4 \pm 10.3$ & 0.7354 \\
\hline Acute coronary syndrome & 21 & 0 & 0.000 \\
\hline Heart failure & 9 & 0 & 0.007 \\
\hline Blood glucose & $11.9 \pm 5.6$ & $10.2 \pm 3.5$ & 0.5133 \\
\hline Glycated hemoglobin (HbA1C) & $8.0 \pm 1.8$ & $8.9 \pm 1.7$ & 0.0876 \\
\hline Low-density lipoprotein (LDL) & $2.2 \pm 0.74$ & $2.5 \pm 0.86$ & 0.4542 \\
\hline Total cholesterol & $3.8 \pm .86$ & $4.1 \pm 1.0$ & 0.3536 \\
\hline
\end{tabular}

\section{TABLE 3: Comparison between patients with DM and DPN with or without CVD}

DM: diabetes mellitus; CVD: cardiovascular disease; DPN: diabetic peripheral neuropathy

\section{Discussion}

In the current study, the rate of DPN was significantly higher in the CVD group and specifically in participants diagnosed with ACS. Many of the patients were unaware that they had DPN. We also found that DPN was associated with a higher HbA1c score and a higher level of blood glucose. However, the other risk factors (gender, age, LDL, cholesterol), although numerically higher in the group with DPN, were not statistically significant.

Three studies are available reporting an association between DPN and increased CVD events [5-7]. Although they used different methods to assess DPN, they demonstrated an association between the two DM complications. One of the studies used the same neuropathic disability score (NDS) to assess DPN and reported an association [6]. Another cohort study, with 13,043 patients, reported that DPN was associated with an increased risk of a first CVD event in patients diagnosed with DM [5]. A prospective cohort study investigating DPN as a predictor for developing CVD in DM patients reported that patients with DM and CVD at baseline had an increased risk of developing DPN (Table 4) [7]. 


\section{Cureus}

\begin{tabular}{|c|c|c|c|c|}
\hline First Author & Publication Date & Sample Size & Study Design & Conclusion \\
\hline Dimitrios Baltzis [6] & Aug 23, 2016 & 82 & Cross-sectional & DPN associated with MI \\
\hline Jack RW Brownrigg [5] & Aug 5, 2014 & 13,043 & Cohort & DPN associated with increased CVD risk \\
\hline Juan Ybarra-Muñoz [7] & Dec 19, 2014 & 267 & Cohort & D associated with in \\
\hline
\end{tabular}

\section{TABLE 4: Summary of similar studies}

DM: diabetes mellitus; CVD: cardiovascular disease; DPN: diabetic peripheral neuropathy; MI: myocardial infarction

A limitation of this study was the small sample size. Larger sample size would have enabled us to assess other risk factors for CVD in addition to DPN.

\section{Conclusions}

The rate of DPN in the CVD group was noticeably higher as compared to the non-CVD group. ACS was significantly associated with DPN but HF was not associated. DPN had uncontrolled risk factors and careful clinical assessment might indicate a higher incidence than what is currently known.

\section{Additional Information \\ Disclosures}

Human subjects: All authors have confirmed that this study did not involve human participants or tissue. Animal subjects: All authors have confirmed that this study did not involve animal subjects or tissue. Conflicts of interest: In compliance with the ICMJE uniform disclosure form, all authors declare the following: Payment/services info: All authors have declared that no financial support was received from any organization for the submitted work. Financial relationships: All authors have declared that they have no financial relationships at present or within the previous three years with any organizations that might have an interest in the submitted work. Other relationships: All authors have declared that there are no other relationships or activities that could appear to have influenced the submitted work.

\section{References}

1. The country report, the IDF Atlas 2015 - Saudi Arabia. International Diabetes Federation . (2015). Accessed: December 13, 2016: http://reports.instantatlas.com/report/view/846e76122b5f476fa6ef09471965aedd/SAU.

2. Diabetes country profile. Saudi Arabia. WHO. (2016). Accessed: December 13, 2016 : http://www.who.int/diabetes/country-profiles/sau_en.pdf?ua=1.

3. Boulton A, Malik R, Arezzo J, Sosenko J: Diabetic somatic neuropathies. Diabetes Care. 2004, 27:1458-1486. 10.2337/DIACARE.27.6.1458

4. Thompson A, Di Angelantonio E, Gao P, Sarwar N: Diabetes mellitus, fasting glucose, and risk of causespecific death. N Engl J Med. 2011, 13:829-841. 10.1056/NEJMoa1008862

5. Brownrigg J, de Lusignan S, McGovern A, et al.: Peripheral neuropathy and the risk of cardiovascular events in type 2 diabetes mellitus. Heart. 2014, 13:1837-1843. 10.1136/heartjnl-2014-305657

6. Baltzis D, Roustit M, Grammatikopoulou M, et al.: Diabetic peripheral neuropathy as a predictor of asymptomatic myocardial ischemia in type 2 diabetes mellitus: a cross-sectional study. Adv Ther. 2016, 33:1840-1847. 10.1007/s12325-016-0399-1

7. Ybarra-Muñoz J, Jurado-Campos J, Garcia-Gil M, et al.: Cardiovascular disease predicts diabetic peripheral polyneuropathy in subjects with type 2 diabetes: a 10-year prospective study. Eur J Cardiovasc Nurs. 2014, 11:248-254. 10.1177/1474515114565215 